\title{
Ocular immunity and peculiarities around COVID-19: Can universal protective eye wear be a game changer?
}

\author{
Mohamed AW Hussein ${ }^{1 *}$ and Eiman Hussein ${ }^{2}$ \\ ${ }^{1}$ Department of Ophthalmology, Texas Children's Hospital and Baylor College of Medicine, Houston, Texas, United States \\ ${ }^{2}$ Department of Clinical Pathology, Faculty of Medicine, Cairo University, Cairo, Egypt
}

\begin{abstract}
This article describes a possible role that the ocular immunity may play in determining the severity of COVID-19 infection and in explaining most of the peculiarities around COVID-19. The article also describes the possibility of the use of cyclosporin eye drops in the prophylaxis as well as the treatment of patients with COVID-19.
\end{abstract}

\section{To the editor,}

One of the interesting phenomena that has been observed with COVID-19 is the fact children, particularly those younger than 10 years of age, seem to be less affected in terms of COVID-19 severity and possibly also frequency (CDC, April 10, 2020). This is counterintuitive to most other respiratory viral infections which tend to affect children at a higher frequency and with greater severity. Additional interesting observation around COVID-19 is the fact that the severity of infections and the mortality of the disease seem to be less in less developed countries. Much of the research centered around finding an explanation for these peculiarities focuses on looking into host factors. Looking into the route of transmission for this virus, as a possible explanation for the aforementioned observations, has not been considered. Ocular involvement was thought to play a less important role; however, recent data suggests a larger role of the eye in transmission of COVID-19. The virus affinity to the conjunctival epithelium was found to be as high as its affinity to the epithelial lining of the respiratory tract [1]. Data also suggested that the virus can replicate aggressively and for a long time in the ocular fluid [2]. Additionally, another study revealed that up to $30 \%$ of COVID-19 cases had ocular manifestations, which were typically seen in patients with more severe disease [3].

The above data suggests that ocular involvement may play a more important role in the transmission of COVID-19. Moreover, ocular infection can potentially be a factor determining the severity of the disease. The later suggestion is based on the ability of ocular immunity to explain all the peculiarities around COVID-19.

One possible explanation for why children may be less severely affected by this infection compared to adults is the difference in the ocular immune response. Few organs have this phenomenon where the immune response to foreign antigens is weak or suppressed [4]. The eye of adults is one of these immune privileged organs. Children's eyes, on the other hand, are not immune privileged and tend to mount stronger immune response compared to adult eyes, which may halt the progression of the viral infection.

Another discernible observation is the fact that less developed countries have less disease severity as well as mortality. One possible explanation for this observation may be related to the fact that the eyes of some patients in most of these less developed countries may not be as immune privileged as they are in most developed countries because of endemic ocular infections that may enforce the innate ocular immunity and allow the eye to fight infections more reliably. One common endemic ocular infection shared by most under-developed countries, which tends to have less mortality from disease, is trachoma. The map for countries where trachoma is endemic tends to strongly correlate with the map for less mortality from this virus infection [5].

Another interesting observation, that may support more role of the eye in directing the severity of the disease is the fact that most Asian countries were able to control transmission of this virus at faster rates than most other countries; examples include South Korea and Singapore. It is well known that a significant percentage of Asians have high refractive errors and wear corrective glasses [6]. The use of glasses may have helped to slow and control the transmission of the virus. This was further supported in two previous studies $[7,8]$.

The data stated may suggest that the weak innate ocular immune response, typical of adult eyes, may allow the virus to replicate unchecked on the ocular surface and in the tears, allowing a significant dose of the virus to be delivered through the nasolacrimal duct system to the nasopharyngeal area. This may challenge the natural nasopharyngeal immune system resulting in a severe disease.

As such, ocular medications may be an ideal treatment option. Most medications applied to the eye would exert their effect on the ocular surface then drain through the nasolacrimal duct into the nasopharynx, followed by a rapid absorption of a portion of the medication through the vascular nasal mucosa, allowing for systemic effects.

Of all the topical medications, cyclosporine appears to be the one that demonstrated, in cell culture, the ability to stop replication of other

${ }^{\star}$ Correspondence to: Mohamed AW Hussein, MD, 6701 Fannin, St 610.25, Houston, Texas 77030, USA; E-mail: mahussei@texaschildrenshospital.org

Key words: covid-19, cyclosporine, epidemiology, immunology

Received: March 04, 2021; Accepted: March 11, 2021; Published: March 16, 2021 
types of coronaviruses, including SARS CoV, at a very low non-cytotoxic dose of 16 micromolars [9]. One drop of the $0.05 \%$ cyclosporine is calculated to contain approximately 80 micromolars, which is 5 folds the lethal dose to SARS CoV1.

In summary, the possibility of the role the ocular pathway plays as a source of COVID-19 transmission in determining the severity of the disease may need to be closely looked at. Given the evidence in the literature that cyclosporine may help alter the course of coronaviruses similar to COVID-19, clinical trials may be warranted looking into the effectiveness of the use of cyclosporine eye drops in reducing the viral load in healthy patients diagnosed with COVID-19.

\section{Declaration}

\section{Ethics approval and consent to participate}

Not applicable

\section{Consent for publication}

Not applicable

\section{Competing interests}

The authors declared no potential conflicts of interest with respect to the research, authorship, and/or publication of this article.

\section{Funding}

The authors received no financial support for the research, authorship, and/or publication of this article.

\section{Authors' contributions}

MAWH: designed, drafted, critical revised the manuscript
EH: Drafted and critically revised the manuscript.

All authors read and approved the final manuscript.

\section{Acknowledgements}

Not applicable

\section{References}

1. Hui KPY, Cheung MC, Perera RAPM, Ng Ka-Chun, Bui CHT, et al. (2020) Tropism, replication competence, and innate immune responses of the coronavirus SARS-CoV-2 in human respiratory tract and conjunctiva: an analysis in ex-vivo and in-vitro cultures. Lancet Respir Med 8: 687-695.

2. Colavita F, Lapa D, Carletti F, Lalle E, Bordi L, et al. (2020) SARS-CoV-2 isolation from ocular secretions of a patient with COVID-19 in Italy with prolonged viral RNA detection. Ann Intern Med 173: 242-243.

3. Wu P, Duan F, Luo C, Liu Q, Qu X, et al. (2020). Characteristics of ocular findings of patients with Coronavirus Disease 2019 (COVID-19) in Hubei Province, China. JAMA Ophthalmol 138: 575-578.

4. Forrester JV, Xu H (2012) Good news-bad news: The Yin and Yang of immune privilege in the eye. Front Immunol 3: 338. [Crossref]

5. Polack S, Brooker S, Kuper H, Mariotti S, Mabey D, et al. (2005) Mapping the global distribution of trachoma. Bull World Health Organ 83: 913-919.

6. Rudnicka AR, Kapetanakis VV, Wathern AK, Logan NS, Gilmartin B, et al. (2016) Global variations and time trends in the prevalence of childhood myopia, a systematic review and quantitative meta-analysis: implications for aetiology and early prevention. Br J Ophthalmol 100: 882-890. [Crossref]

7. Weibiao Zeng, Xiaolin Wang, Junyu Li, Yang Y, Qiu X, et al. (2020) Association of daily wear of eyeglasses with susceptibility to Coronavirus Disease 2019 Infection. JAMA Ophthalmol 138: 1196-1199. [Crossref]

8. Raboud J, Shigayeva A, McGeer A, Bontovics E, Chapman M, et al. (2010) Risk factors for SARS transmission from patients requiring intubation: a multicentre investigation in Toronto, Canada. Plos One 5: e10717. [Crossref]

9. de Wilde AH, Zevenhoven-Dobbe JC, van der Meer Y, Thiel V, Narayanan K, et al (2011) Cyclosporin A inhibits the replication of diverse coronaviruses. J Gen Virol 92: 2542-2548. [Crossref] 